DOI: 10.12957/demetra.2018.29559

\title{
Perfil lipídico e glicêmico de ratos submetidos a dietas com fibra da casca e talo de banana
}

\section{Lipid and glycemic profile of rats fed on diets with banana peel and banana stalk}

\author{
Thielen Borba da Costa' \\ Catia Silva Silveira' \\ Marina Couto Pereiral \\ Elizabete Helbig'
1 Universidade Federal de Pelotas, Faculdade de Nutrição. Pelotas, RS, Brasil.
Correspondência / Correspondence
Thielen Borba da Costa
E-mail: thielenborba@hotmail.com

\section{Resumo}

Objetivo: O estudo teve por objetivo avaliar os efeitos da ingestão de dietas contendo a fibra presente na casca e talo da banana, no perfil glicêmico e lipídico de ratos da linhagem Wistar. Metodologia: Estudou-se, durante 65 dias, a influência de dietas contendo fibras provenientes da casca (GFC), talo da banana (GFT) e ambas as fibras (GFCT), em relação à dieta controle (GA), no perfil glicêmico e lipídico de 24 ratos machos da linhagem Wistar, com peso médio inicial de 54,06g, distribuídos de forma equitativa em quatro grupos. O grupo controle (GA) recebeu dieta AIN acrescida de $1 \%$ de colesterol cristalino e $0,1 \%$ de ácido cólico, e as dietas experimentais foram semelhantes ao grupo controle, com substituição da fonte de fibra. Resultados: Nos grupos de tratamento (GFC, GFT e GFCT), houve aumento dos níveis glicêmicos, de triglicerídeos e redução do colesterol total e LDL-C séricos, em relação ao grupo controle (GA). Os níveis de VLDL-C mostraram-se reduzidos apenas para o grupo contendo fibras provenientes da casca. Conclusões: A inserção de fibra na dieta, proveniente da casca e talo da banana, bem como da mistura de ambos, mostrou efetividade de uso sobre o perfil lipídico e capacidade de manutenção dos níveis de glicemia dos ratos alimentados com dietas contendo fibra oriunda da casca e do talo.

Palavras-chave: Fibra Alimentar. Banana. Glicemia. Ratos. Lipídeos. 


\section{Abstract}

Objective: The aim of this study was to evaluate the effects of dietary intake of fiber present in banana peel and stalk on the blood glucose and lipid profiles of Wistar rats. Methodology: This research was conducted for 65 days to study the influence of diets containing fiber from banana peel (PGF), banana stalks (SFG) and both types of fiber (PSFG), as compared with the control diet (CG), on the glycemic and lipid profile of 24 male Wistar rats, with a mean initial weight of $54.06 \mathrm{~g}$, distributed equally into four groups. The control group (CG) received the AIN diet plus $1 \%$ crystalline cholesterol and $0.1 \%$ colic acid and the experimental diets were similar to the control group, except for substitution of the fiber source. Results: In the treatment groups (PFG, SFG and PSFG), there was an increase in glycemic, triglyceride and serum total cholesterol and LDL-C levels compared with the control group (CG). The levels of VLDL-C were reduced only in the group containing fibers from the peel. Conclusions: Inclusion of dietary fiber from banana peels and stalks, as well as the mixture of both types of fiber, showed effectiveness of use on the lipid profile and the ability to maintain the blood glucose levels of rats fed on diets containing fiber from the peel and the stalk.

Keywords: Dietary Fiber. Banana. Glycemia. Rats. Lipids.

\section{Introdução}

Na tentativa de adequar a alimentação ao ritmo acelerado do dia a dia, as escolhas e os hábitos de consumo tiveram de se adaptar a um novo estilo de vida. A população, dessa forma, vem optando pelo consumo de alimentos industrializados, que são rápidos e práticos e possuem baixo aporte nutricional e elevado valor calórico, decorrente da presença do excesso de açúcar e gordura. ${ }^{1}$ Segundo Martins et al., ${ }^{2}$ os brasileiros, em pouco mais de vinte anos, entre 1987 e 2009 , aumentaram em mais de $10 \%$ o consumo de alimentos ultraprocessados e quase $12 \%$ a ingestão de alimentos prontos para consumo.

É sabido que o consumo desses alimentos em grande escala contribui para o aumento significativo da prevalência e incidência de doenças crônicas não transmissíveis (DCNT), ${ }^{3}$ a exemplo das doenças cardiovasculares, muitas vezes causadas por dislipidemias, que causam alterações dos níveis de lipídios circulantes ${ }^{4}$ e o diabetes, definido pelas Diretrizes da Sociedade Brasileira de Diabetes ${ }^{5}$ como um grupo de distintos distúrbios metabólicos que apresentam em comum a 
hiperglicemia, resultante de defeitos na ação da insulina e/ou na sua secreção. Essas patologias contribuem substancialmente com o aumento do número de óbitos e da morbidade, causadas por DCNT entre adultos e idosos no Brasil. ${ }^{3}$

De acordo com Schmidt et al., ${ }^{6} 72 \%$ das mortes no Brasil em 2007 foram atribuídas as DCNT e 74\% em 2012, conforme a WHO. ${ }^{7}$ As doenças cardiovasculares em conjunto com outras DCNT, entre elas o diabetes, no Brasil, representaram 55,2\% do total de causas de óbito. ${ }^{3}$ Dados brasileiros de 2011 mostram que as taxas de mortalidade por diabetes são de 33,7/100 mil habitantes, para a população geral, ${ }^{8}$ e que algum tipo de dislipidemia foi verificado em $59,74 \%$ da população do município de São Paulo em 2008, conforme estudos realizados por Garcez et al. ${ }^{9}$

O Brasil é a segunda sub-região (entre oito), de acordo com a divisão da Organização Panamericana da Saúde (PAHO), ${ }^{10}$ a apresentar maior probabilidade de alguém com 30 anos de idade ser portador de alguma das principais DCNT, como diabetes, câncer, doença crônica respiratória e doença cardiovascular.

Algumas ações devem ser praticadas pelos indivíduos que buscam a prevenção e/ou tratamento destas doenças, e dentre elas está o tratamento dietético, através da alimentação nutricionalmente balanceada, composta de alimentos como cereais integrais, frutas e legumes. Tais grupos são boas fontes de fibras alimentares, as quais desempenham papel importante na prevenção e tratamento de DCNT, não só da diabetes e dislipidemias, mas também das doenças cardiovasculares, neoplasias, constipação e obesidade. ${ }^{11-13}$

As fibras alimentares são caracterizadas como um conjunto de substâncias derivadas de vegetais resistentes à ação das enzimas digestivas humanas. ${ }^{14}$ Já na $30^{\underline{a}}$ reunião do CodexAlimentarius, ${ }^{15}$ definiu-se fibra alimentar como aquela constituída de polímeros de carboidratos, com dez ou mais unidades monoméricas, que não são hidrolisados pelas enzimas endógenas no intestino delgado e que podem pertencer a três categorias: (i) polímeros de carboidratos comestíveis; (ii) polímeros de carboidratos obtidos de material cru por meio físico, químico ou enzimático; e (iii) polímeros de carboidratos sintéticos.

Quanto à recomendação de ingestão de fibra alimentar total para adultos, estabeleceram-se $25 \mathrm{~g} / \mathrm{dia}$ para mulheres e $30 \mathrm{~g} / \mathrm{dia}$ para homens, sendo que para pacientes com diabetes, a ingestão diária recomendada é de $14 \mathrm{~g}$ de fibras a cada $1.000 \mathrm{kcal}$ ingeridas. ${ }^{16}$

A $5^{\text {a }}$ Diretriz Brasileira de Dislipidemias e Prevenção da Aterosclerose reforça a magnitude e a evidência de que o consumo de fibras solúveis está associado à redução do LDL-C e colesterol total. Outra consideração é que a ingestão adequada desse tipo de fibra é fator importante contra o surgimento do diabetes, por ele ser capaz de se ligar aos nutrientes no trato gastrointestinal, tornando a absorção de glicose mais lenta. ${ }^{17}$ 
Em virtude dos argumentos apresentados, verifica-se a importância do consumo de produtos alimentícios ricos em fibra, a exemplo de subprodutos como cascas, talos e folhas, que, segundo David, ${ }^{18}$ representa $30 \%$ do desperdício de alimentos hortifrutigranjeiros comprados, devido à falta de conhecimento sobre seu valor nutricional ou forma de preparo adequada.

De acordo com Stork et al., ${ }^{19}$ as partes não aproveitáveis dos alimentos, como por exemplo o talo, podem ser utilizadas para aumentar o valor nutricional de uma refeição, já que podem ser mais nutritivos do que a parte nobre do alimento.

No Brasil, a produção de banana, em 2013, foi de sete milhões de toneladas, colocando o país como o quinto maior produtor mundial da fruta. ${ }^{20}$ Trata-se do fruto da bananeira (Musa sp.), uma árvore frutífera tropical, encontrada em todo o mundo. ${ }^{21}$ No estudo de Gondim et al., ${ }^{22}$ evidenciouse que as maiores quantidades de fibras foram encontradas na casca, quando comparada à parte comestível in natura.

Tendo em vista o aproveitamento de forma integral dos alimentos e que a fibra alimentar tem papel importante na digestão alimentar e prevenção de doenças, o presente estudo teve como objetivo avaliar os efeitos da ingestão de dietas contendo a fibra presente na casca e talo da banana, no perfil glicêmico e lipídico de ratos da linhagem Wistar.

\section{Metodologia}

\section{Caracterização do local e matéria-prima}

O ensaio biológico foi realizado no Laboratório de Nutrição Experimental da Faculdade de Nutrição da Universidade Federal de Pelotas (UFPel), em Pelotas-RS. Os talos e cascas de banana prata foram fornecidos por um estabelecimento comercial da cidade de Pelotas-RS.

\section{Métodos Analíticos}

Para obtenção da fibra presente nos talos e nas cascas, foram adaptados os procedimentos de Beltran, ${ }^{23}$ utilizando os talos e cascas de banana prata. A matéria-prima foi lavada em água corrente durante 15 minutos, a fim de retirar as possíveis sujeiras. Após a seleção dos talos e cascas não danificados, os mesmos foram autoclavados durante 30 minutos a uma pressão de $1,5 \mathrm{kgf} / \mathrm{cm}^{2}$ a 121ํㅡ. As fibras do material autoclavado foram trituradas em um liquidificador doméstico, com posterior secagem em estufa a $100^{\circ} \mathrm{C}$ durante 24 horas. $\mathrm{O}$ material obtido foi misturado à dieta AIN-93G na substituição da fibra em quantidade equivalente entre as dietas. 
No final do período experimental, sob supervisão de um médico veterinário, os ratos, após jejum de 12 horas, foram anestesiados e posteriormente decapitados, com o uso de guilhotina, para coleta de sangue. Todo o procedimento foi realizado de acordo com a Resolução do Conselho da Faculdade de Medicina Veterinária, ${ }^{24}$ seguindo os Princípios Éticos na Experimentação Animal, adotados pelo Colégio Brasileiro de Experimentação Animal $(\mathrm{COBEA})^{25}$ e aprovados pela Comissão de Ética em Experimentação Animal (CEEA) ${ }^{26}$, sob o número 23110.004536/2015-45.

Para a avaliação da glicemia, o sangue coletado foi centrifugado por $3500 \mathrm{rpm}$, a temperatura ambiente, por dez minutos, para se obter o plasma sanguíneo. Para aferição das concentrações plasmáticas, as mesmas foram dosadas e expressas em $\mathrm{mG} / \mathrm{dL}$, por kit colorimétrico da marca Bioclin $^{\circledR}$.

Já para a análise do perfil lipídico, o sangue coletado foi centrifugado por $3500 \mathrm{rpm}$ a temperatura ambiente, por 10 minutos, para se obter o soro sanguíneo, que foi congelado e armazenado a $-20^{\circ} \mathrm{C}$ até a análise das frações lipídicas.

O colesterol sérico foi quantificado por sistema enzimático (colesterol esterase, colesterol oxidase e peroxidase Labtest Diagnóstica ${ }^{\circledR}$ colesterol liquiform cat. 76-2/100).

Para determinação do VLDL, foi utilizada a fórmula VLDL - Triacilglicerol/5 e o LDL pela diferença de colesterol total e (HDL + VLDL), conforme descrito por Friedwal et al. ${ }^{27}$ Os triacilgliceróis (TGL) foram determinados pelo sistema enzimático Labtest Diagnostica ${ }^{\circledR}$ (GPO_ANA cat. 59-4/50).

\section{Métodos Biológicos}

No ensaio biológico, foram utilizados 24 ratos machos da linhagem Wistar (Rattus norvegicus), em crescimento, provenientes do Biotério Central da UFPel, com peso médio de 54,06g $\pm 4,13$.

Os animais foram mantidos em gaiolas metabólicas individuais, em gabinete ventilado, com temperatura e umidade relativa de respectivamente $22^{\circ} \mathrm{C} \pm 24^{\circ} \mathrm{C}$ e $65-75 \%$, ciclos de claro/escuro de 12 horas com água e ração ad libitum. O ensaio biológico teve duração de 65 dias, sendo que os primeiros cinco dias foram de adaptação à dieta.

Para a condução do experimento, foram adotadas as recomendações de Reeves et al., ${ }^{28} \mathrm{com}$ algumas modificações. Os animais foram distribuídos em blocos casualizados e submetidos a quatro dietas que constituíram os tratamentos experimentais deste estudo. A dieta AIN-93G modificada foi utilizada no grupo controle (GA), contendo $1 \%$ de colesterol e $0,1 \%$ de ácido cólico, e os demais grupos receberam dieta AING-93 modificada quanto à fonte de fibra: fibra da casca da banana (GFC), fibra do talo (GFT) e ambas as fibras (GFCT), sendo que todas as dietas continham $1 \%$ de colesterol e $0,1 \%$ de ácido cólico. 
A partir de 59 dias, fase de manutenção de peso, os ratos passaram a consumir as dietas formuladas segundo a AIN-93M, mantendo os percentuais anteriormente indicados para as fibras, colesterol e ácido cólico.

\section{Análise Estatística}

Os resultados foram expressos em função da média e o desvio padrão, e estatisticamente avaliados. Utilizando-se análise de variância (ANOVA), as diferenças significativas entre médias $(\mathrm{p}<0,05)$ foram estabelecidas pelo Teste estatístico de Tukey. Para as análises, utilizaram-se o aplicativo Microsoft Excel 2013 e o software Statistica versão 11.0.

\section{Resultados}

Na tabela 1, são apresentadas as concentrações médias de colesterol total, VLDL-C, LDL-C, triacilgliceróis (TGL) e glicose no soro de ratos após 60 dias de consumo das dietas experimentais.

A partir dos resultados obtidos, evidenciou-se que houve redução significativa $(\mathrm{p}<0,05)$ do colesterol sérico, para os animais alimentados com as dietas contendo como fonte de fibra a casca - GFC $\left(140,95 \pm 0,127 \mathrm{mg} / \mathrm{dL}^{-1}\right)$ e talo - GFT $\left(105,37 \pm 0,12 \mathrm{mg} / \mathrm{dL}^{-1}\right)$, sendo que para este último observou-se redução de 47,5\% dos níveis, quando comparado ao grupo com dieta controle - GA $\left(200,95 \pm 0,11 \mathrm{mg} / \mathrm{dL}^{-1}\right)$.

Para a LDL-C, foi observada diminuição significativamente representativa $(\mathrm{p}<0,05)$ para os ratos alimentados com dieta cuja fonte de fibra foram os talos - GFT $\left(28,43 \pm 3,93 \mathrm{mg} / \mathrm{dL}^{-1}\right)$, quando comparados aos resultados com o grupo com dieta controle - GA $\left(200,95 \pm 0,11 \mathrm{mg} / \mathrm{dL}^{-1}\right)$. No que se refere às VLDL-C e ao TGL, não foram identificadas diferenças estatísticas em relação ao grupo controle.

Cabe considerar que, em relação aos níveis de VLDL-C, apenas em GFC se encontrou uma média dos níveis séricos menores que os encontrados no grupo controle (GA), porém não foi constatada nenhuma diferença estatística $(\mathrm{p}>0,05)$ nos níveis séricos de nenhum dos grupos estudados. Resultado equivalente foi encontrado ao analisar os níveis de TGL.

Verificou-se, na tabela 1 , que em relação à glicemia, não houve diferença significativa $(\mathrm{p}>0,05)$ dos níveis séricos, dos submetidos às dietas GFC e GFT, quando comparados aos submetidos a dieta GA. No entanto, observou-se aumento significativo dos níveis glicêmicos para os alimentados com a dieta GFCT. 
Tabela 1. Perfil lipídico e glicêmico do soro sanguíneo $(\mathrm{mg} / \mathrm{dl})$ de ratos alimentados com dietas experimentais contendo diferentes fontes de fibra (média \pm desvio padrão). Pelotas-RS, 2016.

\begin{tabular}{cccccc}
\hline Grupo & $\begin{array}{c}\text { CT } \\
\left(\mathrm{mg} / \mathrm{dL}^{-1}\right)\end{array}$ & $\begin{array}{c}\text { LDL-C } \\
\left(\mathrm{mg} / \mathrm{dL}^{-1}\right)\end{array}$ & $\begin{array}{c}\text { VLDL-C } \\
\left(\mathrm{mg} / \mathrm{dL}^{-1}\right)\end{array}$ & $\begin{array}{c}\text { TGL } \\
\left(\mathrm{mg} / \mathrm{dL}^{-1}\right)\end{array}$ & $\begin{array}{c}\text { Glicose } \\
\left(\mathrm{mg} / \mathrm{dL}^{-1}\right)\end{array}$ \\
\hline GA & $200 \pm 0,11^{\mathrm{a}}$ & $62 \pm 8,74^{\mathrm{a}}$ & $13,56 \pm 0,85^{\mathrm{a}}$ & $67,81 \pm 0,11^{\mathrm{a}}$ & $100 \pm 0,019^{\mathrm{a}}$ \\
GFC & $140,95 \pm 0,127^{\mathrm{bc}}$ & $51,73 \pm 11,32^{\mathrm{a}}$ & $12,54 \pm 2,86^{\mathrm{a}}$ & $62,72 \pm 0,13^{\mathrm{a}}$ & $109,97 \pm 0,014^{\mathrm{a}}$ \\
GFT & $105,37 \pm 0,12^{\mathrm{c}}$ & $28,43 \pm 3,93^{\mathrm{b}}$ & $14,40 \pm 2,04^{\mathrm{a}}$ & $72,01 \pm 0,14^{\mathrm{a}}$ & $121,20 \pm 0,04^{\mathrm{a}}$ \\
GFCT & $167,59 \pm 0,16^{\mathrm{ab}}$ & $37,27 \pm 12,17^{\mathrm{a}}$ & $15,59 \pm 3,03^{\mathrm{a}}$ & $76,50 \pm 0,20^{\mathrm{a}}$ & $163,69 \pm 0,02^{\mathrm{b}}$ \\
\hline
\end{tabular}

Médias nas colunas seguidas por letras diferem entre si ao nível de significância de 5\% pelo teste de Tukey. GA: Grupo AIN (Controle); GFC: Grupo Casca; GFT: Grupo Talo; GFCT: Grupo Mista; CT: Colesterol total; VLDL-C: VLDL-colesterol; LDL-C: LDL-colesterol; TGL: Triacilgliceróis.

\section{Discussão}

No que se refere ao colesterol total, os resultados observados evidenciaram que a utilização de dietas GFC e GFT, contendo fibra solúvel (pectina), mostraram efetividade estatística $(\mathrm{p}<0,05)$ em relação aos alimentados com a dieta GA, que continha fibra insolúvel (celulose). Este resultado está em concordância com os achados de Fietz \& Salgado, ${ }^{29}$ que verificaram redução mais expressiva dos níveis séricos de colesterol de ratos da linhagem Wistar alimentados durante 30 dias com dieta contendo pectina, quando comparados aos que consumiram celulose.

Já Barroso et al., ${ }^{30} \mathrm{em}$ seu estudo com dietas à base de fibras do talo de couve e espinafre, ambas com predominância de fibra insolúvel, encontraram grande redução na média de triacilglicerol sérico. Sales et al., ${ }^{31}$ em sua pesquisa, verificaram que os níveis de LDL-C e TGL em ratos diabéticos em tratamento com alimentação enriquecida com aveia, linhaça, gergelim e girassol, apesar de se elevarem ao fim do experimento, não foram tão altos quanto aqueles animais diabéticos que recebiam dieta controle por 50 dias.

Esses resultados confirmam que a viscosidade é a principal propriedade da fibra, capaz de reduzir os níveis de colesterol e LDL-C séricos. Isto ocorre devido à excreção de ácidos biliares nas fezes, que acaba provocando a conversão de colesterol do sangue para esses ácidos. ${ }^{32}$

A relação entre os valores equivalentes de VLDL-C e TGL, nos grupos de tratamento, é possível de ser explicada, uma vez que essas lipoproteínas (VLDL) são as principais transportadoras de TGL no plasma. ${ }^{33}$

No que se refere à glicemia, o tipo de fibra e a ação fisiológica devem ser considerados. Nos subprodutos da bananeira, tanto na casca como talo da banana, encontram-se fibras solúveis e 
insolúveis. Em relação à casca, em estudo realizado por Rebello, ${ }^{34}$ identificou-se que o teor de fibras insolúveis foi $83,41 \%$, maior que o quantitativo de fibras solúveis. Esta qualidade diferenciada de fibras alimentares, do ponto de vista fisiológico, leva a uma utilização distinta dos nutrientes. No caso, a presença de fibras solúveis no intestino delgado dificulta a ação das enzimas hidrolíticas, o que pode impedir a absorção de nutrientes, bem como retardar a digestão. ${ }^{35}$ Já no caso de fibras insolúveis, conforme descrito pelos mesmos autores, há possibilidade de redução da atividade das enzimas digestivas, como lipase e tripsina, quimiotripsina e amilase.

A partir destas considerações, verifica-se que embora não se tenha observado diferença significativa quanto à glicemia dos alimentados com as dietas casca (GFC) e talo (GFT) em relação aos da dieta controle (GA), identificou-se aumento desta variável para ambos os grupos. Porém, os alimentados com a dieta mista (GFCT) mostraram aumento significativo da glicemia, quando comparados ao grupo controle (GA). Isso pressupõe que os efeitos fisiológicos da mistura das duas fibras tenha sido potencializado, ou seja, diminuído a ação das enzimas digestivas - neste caso, da amilase - culminando com o aumento considerável da glicemia.

\section{Conclusões}

A ingestão de fibras alimentares provenientes da casca e do talo de banana na dieta deve ser incentivada como parte da mudança para novos hábitos saudáveis.

Neste estudo, conclui-se que tais fibras são capazes de regular o perfil lipídico e manter a glicemia de ratos, quando as fibras da casca e talo da banana são utilizadas em separado.

\section{Colaboradores}

Costa TB, Silveira CS, Pereira MC e Helbig E participaram de todas as etapas, desde a concepção do estudo até a revisão da versão final do artigo.

Conflito de Interesses: Os autores declaram não haver conflito de interesses.

\section{Referências}

1. Louzada MLC, Martins APB, Canella DS, Baraldi LG, Levy RB, Claro RM, et al. Alimentos ultraprocessados e perfil nutricional da dieta no Brasil. Rev Saúde Pública 2015; 49(38):1-11.

2. Martins APB, Levy RB, Claro R M, Moubarac JC, Monteiro CA. Increased contribution of ultraprocessed food products in the Brazilian diet (1987-2009). Rev Saúde Pública 2013; 47(4):656-665. 
3. Brasil. Ministério da Saúde. Inquérito domiciliar sobre comportamentos de risco e morbidade referida de doenças e agravos não transmissíveis: Brasil, 15 capitais e Distrito Federal, 2002-2003. Rio de Janeiro: INCA; 2004.

4. Hossri CAC, Silva CC. Bioimpedância Elétrica e calorimetria indireta na avaliação nutricional de portadores de Insuficiência Cardíaca. In: Cukier C, Magnoni D, Alvarez T. Nutrição baseada na fisiologia dos órgãos e sistemas. São Paulo: Sarvier; 2005. p. 100-107.

5. Vencio JEPO, organizador. Diretrizes da Sociedade Brasileira de Diabetes (2015-2016). São Paulo: A.C. Farmacêutica; 2016.

6. Schmidt MI, Duncan BB, Silva GA, Menezes AM, Monteiro CA, Barreto SM, et al. Health in 145 Brazil 4. Chronic non-communicable diseases in Brazil: burden and current challenges. The Lancet 2011; 377(9781):1949-1961.

7. Brazil. In: World Health Organization. Mortality and burden of disease. Noncommunicable Diseases (NCD) country profiles, 2014 [Internet]. Acesso em: dez. 2016. Disponível em: http://www.who.int/ $\mathrm{nmh} /$ countries/bra_en.pdf?ua=1

8. Malta DC, Moura L, Prado RR, Escalante JC, Schmidt MI, Duncan BB. Mortalidade por doenças crônicas não transmissíveis no Brasil e suas regiões, 200 a 2011. Epidemiol Serv Saúde 2014; 23(4):599-608.

9. Garcez MR, Pereira JL, Fontanelli MM, Marchioni DML, Fisberg RM. Prevalência de Dislipidemia segundo estado nutricional em amostra representativa de São Paulo. Arq Bras Cardiol. 2014; 103(6):476-484.

10. Legetic B, Medici A, Hernández-Avila M, Alleyne G, Hennis A, editors. Economic dimensions of non-communicable disease in Latin America and the Caribbean. Disease control priorities. 3. ed. Washington, DC: OPAS; 2016.

11. Bernaud FSR, Rodrigues TC. Fibra alimentar: ingestão adequada e efeitos sobre a saúde do metabolismo. Arq Bras Endocrinol Met. 2013; 57(6):397-405.

12. Aune D, Chan DSM, Lau R, Vieira R, Greenwood DC, Kampman E, et al. Dietary fiber, whole grains, and risk of colorectal cancer: systematic review and dose-response meta-analysis of prospective studies. British Medical Journal 2011; 343:d6617.

13. Anderson JW, Baird P, Davis RH Jr., Ferreri S, Knudtson M, Koraym A, Waters V, Wllims CL. Health benefits of dietary fiber. Nutrition Reviews 2009; 67(4):188-205.

14. Mattos IL, Martins IS. Consumo de fibras alimentares em população adulta. Rev Saúde Pública 2000; 34(1):50-55.

15. World Health Organization. Codex Alimentarius. Report of the 30th Session of the Codex Committee on Nutrition and Foods for Special Dietary Uses; 3-7 Nov. 2008; Cape Town, South Africa.

16. American Diabetes Association. Nutrition recommendations and interventions for diabetes: a position statement of the American Diabetes Association. Diabetes Care 2013; 36(suppl. 1):11-66.

17. Whitney E, Rolfes SR. Nutrição 1: entendendo os nutrientes. São Paulo: Cengage Learning; 2008. 342 p. 
18. David DCZP. Comparação da qualidade bioquímica de vegetais, cultivados de modo convencional e não convencional [Tese]. [Botucatu]: Universidade Estadual Paulista, Instituto de Biociência; 2007. 62 f.

19. Stork CR, Nunes GL, Oliveira BB, Basso C. Folhas, talos, cascas e sementes de vegetais: composição nutricional, aproveitamento na alimentação e análise sensorial de preparações. Ciência Rural 2013; 43(3):537-543.

20. Instituto Brasileiro de Geografia e Estatística. Levantamento sistemático da produção agrícola. Pesquisa mensal de previsão e acompanhamento das safras agrícolas no ano civil. Rio de Janeiro: IBGE; 2017. xx,81 p.

21. Figueiredo FP, Mantovani EC, Soares AA, Costa LC, Ramos MM, Oliveira FG. Produtividade e qualidade da Banana prata anã, influenciada por lâminas de água, cultivada no Norte de Minas Gerais. Rev Bras Eng Agríc Ambient. 2006; 10(4):798-803.

22. Gondim JAM, Moura MFV, Dantas AS, Medeiros RLS, Santos KM. Composição centesimal e de minerais em cascas de frutas. Ciênc Tecnol Aliment. 2005; 25(4):825-827.

23. Beltran JER. Uso da polpa da casca da banana prata na formação de placas isolantes. Pelotas [Dissertação]. [Pelotas]: Universidade Federal de Pelotas; 2014.

24. Brasil. Conselho Federal de Medicina Veterinária. Resolução no 714 de 20 de junho de 2002 [Internet]. acesso em: 30 out. 2015. Disponível em: http://portal.cfmv.gov.br/portal/lei/index/id/327

25. Colégio Brasileiro de Experimentação Animal. Princípios éticos na experimentação animal [Internet]. [acesso em: 21 abr. 2016]. Disponível em: http://www.feis.unesp.br/Home/comissaodeeticaeusoanimal/ principios-eticos-na-experimentacao-animal.pdf

26. Universidade Federal de Pelotas. Comissão de Ética em Experimentação Animal. Regimento da comissão de ética em experimentação animal da Universidade Federal de Pelotas [Internet]. Acesso em: 21 abr. 2016. Disponível em: http://wp.ufpel.edu.br/ceea/regimento/

27. Friedwald WT, Levy RI, Fredrickson DS. Estimation of the concentration of low density lipoprotein cholesterol in plasma, without use of preparative ultracentrifuge. Clin Chem. 1972; 18:499-502.

28. Reeves PG, Nielsen FH, Fahey Jr. GC. AIN-93 Purified diets for laboratory rodents: final report of the American Institute of Nutrition Ad Hoc. Writting Committee on the Reformulation of the AIN-76A Rodent Diet. J Nutr. 1993; 123(11):1939-1951.

29. Fietz VR, Salgado JM. Efeito da pectina e da celulose nos níveis séricos de colesterol e triglicerídeos em ratos hiperlipidêmicos. Ciên Tecnol Aliment. 1999; 19(3):318-321.

30. Barroso AKM, Freitas MCJ, Silva VLM. Avaliação das dietas contendo farinhas de talos de vegetais no trato gastrintestinal e parâmetros bioquímicos de ratos. Nutrire: Rev Soc Bras Aliment Nutr. 2009; 34(3):93-107.

31. Sales ALCC, Teixeira JMR, Soares LFM, Damasceno DCF, Almeida IP, Nunes PHM, et al. Dieta enriquecida em fibras e ácidos graxos polinsaturados: efeitos no controle glicêmico e perfil Lipídico de ratos diabéticos. ARS Veterinária 2010; 26(3):138-146.

32. Colli C, Sardinha F, Filisetti TMCC. Alimentos funcionais. In: Cuppari L. Guia de nutrição: nutrição clínica no adulto. São Paulo: Manole; 2005. p. 71-87. 
33. Silva MAM, Barcelos MFP, Sousa RV, Lima HM, Falco IR, Lima AL, et al. Efeito das fibras dos farelos de trigo e aveia sobre o perfil lipídico no sangue de ratos (Rattus norvegicus) wistar. Ciênc Agrotec. 2003; 27(6):1321-1329.

34. Rebello LPG. Avaliação de compostos fenólicos, extração e caracterização de pectina em farinha de casca de Banana (Musa AAA) [Tese]. [Viçosa]: Universidade Federal de Viçosa; 2013. 87 f.

35. Flora APL, Dichi I. Aspectos atuais na terapia nutricional da doença inflamatória intestina. Rev Bras Nutr Clin. 2006; 21(2):131-137.

Recebido: 13 de julho, 2017

Revisado: 20 de outubro, 2017

Aceito: 03 de janeiro, 2018 
